\title{
Temperature-dependent hardness of zinc-blende structured covalent materials
}

\author{
Xing Feng ${ }^{1}$, Jianwei Xiao ${ }^{1}$, Bin $\mathrm{Wen}^{1^{*}}$, Jijun $\mathrm{Zhao}^{2}$, Bo Xu ${ }^{1^{*}}$, Yanbin Wang ${ }^{3}$ and Yongjun $\operatorname{Tian}^{1}$
}

\begin{abstract}
Understanding the temperature-dependent hardness of covalent materials is of fundamental scientific interest and crucial technical importance. Here we propose a temperature-dependent hardness formula for zinc-blende structured covalent materials based on the dislocation theory. Our results indicate that at low temperatures, the Vickers hardness is primarily modulated by Poisson's ratio and the shear modulus, with the latter playing a dominant role. With an increase in temperature, the governing mechanism for the plastic deformation switches from shuffle-set dislocation control to glide-set dislocation control, and the hardness decreases precipitously at elevated temperatures. Moreover, the intrinsic parameter $a^{3} G$ is revealed for zinc-blende structured covalent materials, which represents the resistance of a material to softening at high temperatures. This temperaturedependent hardness model agrees remarkably well with the experimental data of zinc-blende structured covalent materials. This work not only sheds light on the physical origin of hardness, but also provides a practical guide for the design of superhard materials.
\end{abstract}

Keywords: hardness, temperature effect, dislocation, covalent materials, superhard materials

\section{INTRODUCTION}

Hardness is defined as the ability of a material to resist being dented or scratched by another material [1]. In the past few decades, many studies have been conducted to understand the origin of a material's hardness and determine methods to estimate it. Several hardness models, both macroscopic [1-5] and microscopic [6-9], were established for a wide range of materials with varying degrees of success. For example, using Chen's formula [4], an excellent agreement between the calculated and experimental values of hardness can be achieved for a wide variety of crystalline materials and bulk metallic glasses.

These hardness models were established for ambient conditions where temperature effects were not considered. However, in practical applications, materials are typically operated or processed at variable temperatures where the mechanical behavior and performance are different from those at room temperature. A temperature-dependent hardness model is therefore required. Experimentally, it is challenging to perform temperaturedependent hardness measurements due to the complexities in sample preparation while maintaining a stable temperature during the measurement, which usually results in substantial errors in the measured hardness values. Nonetheless, for zinc-blende structured covalent materials, an intensified softening behavior is clearly observed with increasing temperature [1,10-14]. Using parameters fitted from the experimental data, formulas have been developed to understand this softening behavior $[1,15]$. Still, the fundamental mechanisms, such as the dislocation and microstructure contributions or the effect of loading conditions, behind this temperaturedependent hardness have not been systematically studied, and the applicability to other materials is unknown. Therefore, the establishment of a temperature-dependent hardness model that considers the physical mechanisms is urgently needed, for both scientific and technological reasons.

The difficulty in assessing the hardness, especially with a variable temperature, partially lies in the fact that hardness is an engineering quantity determined using a specific measurement method and cannot be evaluated

\footnotetext{
${ }^{1}$ Center for High Pressure Science, State Key Laboratory of Metastable Materials Science and Technology, Yanshan University, Qinhuangdao 066004, China

${ }^{2}$ Key Laboratory of Materials Modification by Laser, Ion and Electron Beams (Ministry of Education), Dalian University of Technology, Dalian 116024, China

${ }^{3}$ Center for Advanced Radiation Sources, University of Chicago, Chicago, Illinois 60439, USA

* Corresponding authors (emails: wenbin@ysu.edu.cn (Wen B); bxu@ysu.edu.cn (Xu B))
} 
directly using quantum mechanics [16]. During the hardness measurement, plastic deformation must occur in the sample (e.g., a permanent impression or dent), which is correlated with the dislocation behaviors in the sample $[1,17,18]$. While this dislocation-governed plastic deformation has been widely investigated for metals [1922], understanding the plastic deformation and therefore the hardness of covalent materials is still an active research area [23]. For both classes of materials, plastic deformation is closely related to the dislocation glides on the slip systems as well as the microstructure. Our recent work on nanotwinned diamond suggests that roomtemperature ultrahigh hardness can be ascribed to the dislocation behaviors and the nanotwinned microstructure [23-27]. In general, the nucleation and propagation of dislocations in a material can be activated thermally and/or by applied force [28], resulting in a substantial plastic deformation during the hardness evaluation. Therefore, the impact of temperature on hardness can be elucidated by considering the involved dislocations along with other effects, such as the microstructure or the loading conditions. In this work, we re- port a temperature-dependent hardness formula based on the dislocation theory for zinc-blende structured covalent materials.

It is widely known that the hardness and the yield strength of a material both reflect the resistance to plastic deformation, where Tabor's law describes the correlation between hardness and yield strength [29]. Furthermore, the yield strength of a material is directly related to the corresponding critical resolved shear stress (CRSS), as revealed by Schmid's law [28]. Consequently, hardness can be evaluated using CRSS. CRSS is defined as the minimum shear stress required for a dislocation to slip. To understand the temperature-dependent hardness of zinc-blende structured covalent materials (as shown in Fig. 1a), a temperature-dependent CRSS was initially deduced based on the dislocation theory. Due to the strong directional covalent bonds, a large Peierl's barrier is formed when a dislocation moves in covalent materials, restricting the dislocation line to only move along certain directions. These dislocation lines in covalent materials have been observed to propagate primarily through kinks, more specifically through kink pairs [30], as schematically
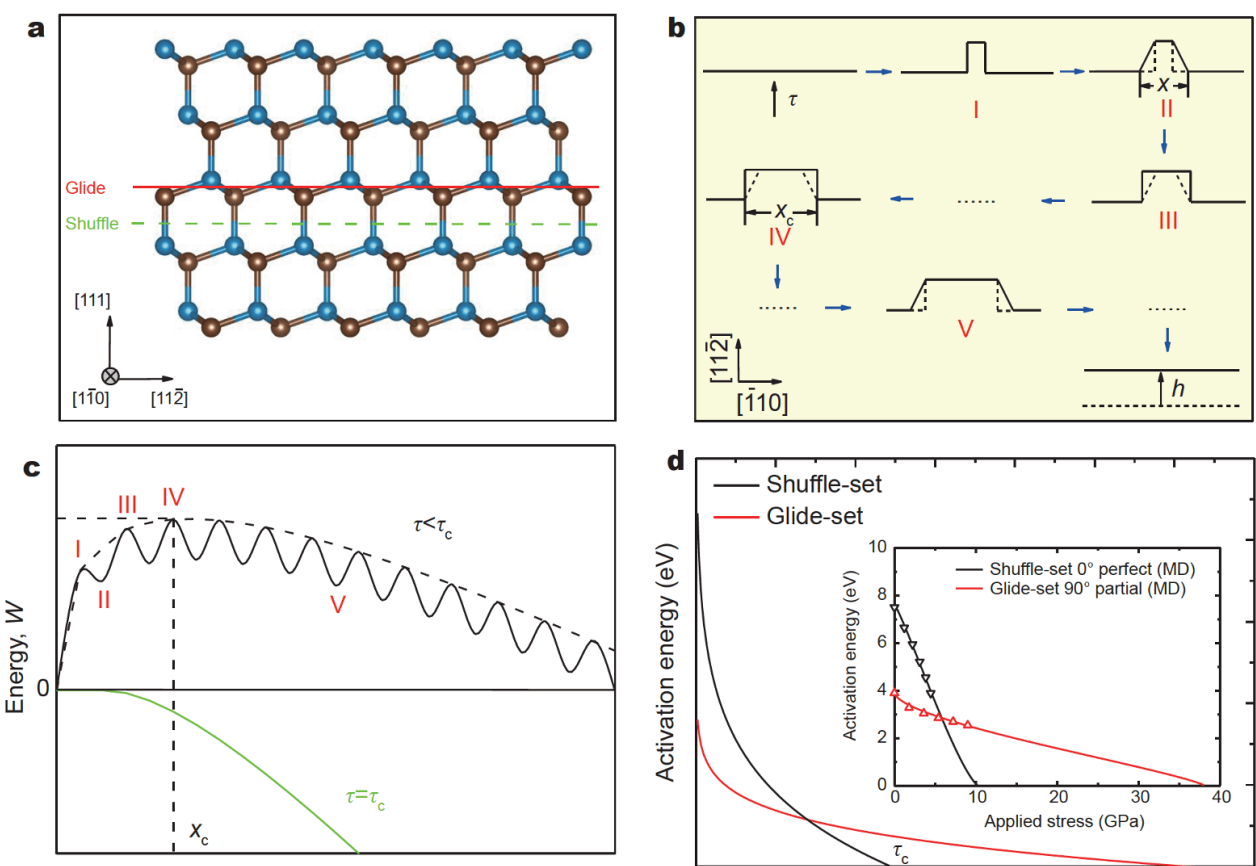

Kink-pair width, $x$

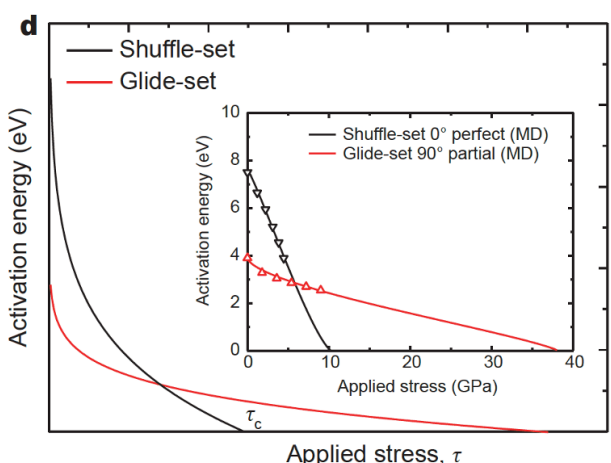

Figure 1 Schematic diagram for the computational method used in this study. (a) $\{110\}$ projection of the zinc-blende structured lattice. The green and red lines indicate the $\{111\}$ shuffle and glide planes, respectively. (b) Kink-pair nucleation and motion process under applied stress. The shear stress acting perpendicular to a dislocation line and parallel to $\langle\overline{1} 10\rangle$ produces a kink pair (I), which subsequently expands (II-V), resulting in an upward motion of the dislocation line along the $\langle 11 \overline{2}\rangle$ direction with a step of $h$. (c) Total energy variation with respect to the kink-pair width under different applied shear stress conditions. The oscillation reflects the lattice periodicity. (d) Activation energy as a function of applied stress for shuffleset and glide-set dislocation motions. The inset shows the MD result for diamond. 
shown in Fig. 1b. Therefore, the CRSS can be obtained by simulating an energy path for a dislocation kink-pair nucleation and the subsequent migration processing. A detailed modeling process is described as follows.

\section{MODEL AND METHODOLOGY}

On the basis of the dislocation theory [28], the total energy $(W)$ of a kink-pair as a function of the kink-pair width $(x)$ and the applied shear stress $(\tau)$ contains four terms: the increment of dislocation elastic energy $\left(W_{\text {elas }}\right)$, the energy of the dislocation core $\left(W_{\text {core }}\right)$, the kink-pair interaction energy $\left(W_{\text {int }}\right)$, and the work done by the applied stress $\left(W_{\tau}\right)$. Usually, $W_{\text {core }}$ is approximately $10 \%-$ $15 \%$ of $W_{\text {elas }}$, so it is ignored in this calculation. Therefore, $W$ can be expressed as

$$
\begin{aligned}
W(x, \tau) & =W_{\text {elas }}+W_{\text {int }}+W_{\tau} \\
& =\frac{A_{1} G b^{2} h}{2 \pi} \ln \left(\frac{R}{r}\right)-\frac{A_{2} G b^{2} h^{2}}{8 \pi x}-h b x \tau,
\end{aligned}
$$

where $b$ is the magnitude of the Burgers vector, $h$ is the kink height, $G$ is the shear modulus, $R$ is the integral range of the linear elasticity theory, $r$ is the radius of the dislocation core, $A_{1}=\cos ^{2} \beta+\frac{\sin ^{2} \beta}{1-v}, \quad$ and $A_{2}=\frac{(1+v) \cos ^{2} \beta+(1-2 v) \sin ^{2} \beta}{1-v}$, where $v$ is Poisson's ratio and $\beta$ is the angle between the Burgers vector and the dislocation line.

As shown in Fig. 1c, $W$ oscillates as a function of $x$ with the lattice periodicity along the $\langle 110\rangle$ direction. The envelope of the local maxima forms a curve (dashed line in Fig. 1c), and the maximum of the envelope can be considered as the activation energy of the dislocation motion. Mathematically, the critical kink-pair width corresponding to the activation energy, $x_{\mathfrak{c}}$, can be determined with the first derivative test [28]. Note that the contribution of $W_{m}$ to the local variation (oscillation) can be ignored when considering the envelope maximum. As a result,

$x_{\mathrm{c}}=\left(\frac{A_{2} h b}{8 \pi} \frac{G}{\tau}\right)^{1 / 2}$,

and the activation energy as a function of $\tau$ can then be determined as

$$
W_{\mathrm{c}}(\tau)=\frac{A_{1} G b^{2} h}{2 \pi} \ln \left(\frac{x_{\mathrm{c}}}{r}\right)-(h b)^{3 / 2}\left(\frac{A_{2} G \tau}{2 \pi}\right)^{1 / 2},
$$

where $R$ in Equation (1) is set to $x_{\mathrm{c}}$ as an approximation.

When considering the temperature effect, the dislocation motion is affected by both the applied shear stress and thermal activation [28]. For a given temperature $T$, applied stress $\tau$, and plastic strain rate $\dot{\varepsilon}$, the temperaturedependent CRSS can be written with a transcendental equation based on Equation (3) and Orowan's relation [28] as the following,

$\tau_{\mathrm{c}}{ }^{T}=\frac{2 \pi}{A_{2} h^{3} b^{3} G}$

$\left[\frac{A_{1} G b^{2} h}{2 \pi} \ln \left(\frac{x_{\mathrm{c}}}{r}\right)-k_{\mathrm{B}} T \ln \left(\frac{\rho_{\mathrm{m}} b \lambda_{\mathrm{b}} v_{\mathrm{D}}}{\dot{\varepsilon}}\right)\right]^{2}$,

where $k_{\mathrm{B}}$ is the Boltzmann constant, $\rho_{\mathrm{m}}$ is the density of mobile dislocations, $\lambda_{\mathrm{b}}$ is the mean free path of dislocations slipping over obstacles, and $v_{\mathrm{D}}$ is the Debye frequency. After substituting Equation (2) into Equation (4), the following is obtained:

$\frac{\tau_{\mathrm{c}}^{T}}{G}=C_{1}\left[C_{2} \ln \left(C_{3} \frac{G}{\tau_{\mathrm{c}}^{T}}\right)-\frac{k_{\mathrm{B}} T}{a^{3} G} \ln \left(\frac{\rho_{\mathrm{m}} b \lambda_{\mathrm{b}} v_{\mathrm{D}}}{\dot{\varepsilon}}\right)\right]^{2}$,

where $a$ is the lattice parameter and $C_{1}=\frac{2 \pi a^{6}}{h^{3} b^{3} A_{2}}$, $C_{2}=\frac{h b^{2} A_{1}}{4 \pi a^{3}}$, and $C_{3}=\frac{h b A_{2}}{8 \pi r^{2}}$ are the dimensionless constants related only to Poisson's ratio of the materials and the geometry of the involved dislocation.

Following Schmid's law, the yield strength is proportional to the CRSS of dislocation as

$\sigma_{y}=m \tau_{c}$,

where $m$ is the Taylor factor.

For covalent materials, the relationship between the yield strength and the Vickers hardness follows Tabor's law, and therefore the Vickers hardness can be expressed as

$H_{v}=\alpha \sigma_{y}$

where $\alpha$ represents the Tabor factor.

Accordingly, a temperature dependent Vickers hardness formula for covalent materials can be expressed as

$H_{T}=m \alpha \tau_{\mathrm{c}}^{T}=n \tau_{\mathrm{c}}^{T}$,

where $n$ is product of the pre-factors in Schmid's law and Tabor's law.

\section{RESULTS AND DISCUSSION}

For zinc-blende structured covalent materials, the main dislocation slip types are $\{111\}<110>[31]$. Due to the two-interpenetrating face-centered crystal (fcc) sublattices, dislocation slips on the $\{111\}$ planes can occur at two different glide planes, i.e., the glide-set and shuffle-set glide planes (Fig. 1a). Based on first-principles calculations (see SI part II for calculation details), the generalized stacking fault energy surfaces were calculated for the 
glide-set and shuffle-set planes in diamond, as shown in Fig. S1. Owing to the existence of a local energy minimum, a $\frac{1}{2}<110>$ glide-set dislocation can be dissociated into two glide-set $\frac{1}{6}<112>$ partial dislocations. In contrast, a $\frac{1}{2}<110>$ shuffle-set dislocation would remain intact due to the excessively high energy required for the dissociation. Meanwhile, the large Peierl's barrier with a deep trough along the $\langle 110\rangle$ direction in zincblende structured materials limits the dislocation lines along these directions [32]. Therefore, two types of dislocations, namely the $\frac{1}{6}<112>$ glide-set $90^{\circ}$ partial (edge) dislocations and the $\frac{1}{2}<110>$ shuffle-set perfect (screw) dislocations, dominate the plastic deformation in zinc-blende structured covalent materials, as indicated in Fig. S2.

The calculated activation energies as a function of the applied shear stress for the $\frac{1}{6}<112>$ glide-set $90^{\circ}$ partial dislocation and the $\frac{1}{2}<110>$ shuffle-set perfect dislocation based on Equation (3) are shown in Fig. 1d. A crossover is clearly indicated at increasing shear stress, indicating a competition between the glide-set and shuffle-set dislocations. This result based on the dislocation theory is further confirmed by molecular dynamics (MD) simulations of diamond (inset of Fig. 1d, also see SI part I for MD calculation detail) and is consistent with previous experimental observations [10].

Table 1 lists the parameters for the $\frac{1}{6}<112>$ glide-set $90^{\circ}$ dislocation and the $\frac{1}{2}<110>$ shuffle-set $0^{\circ}$ perfect dislocation in zinc-blende structured covalent materials. Other values, such as the temperature-dependent lattice constant, shear modulus, and Poisson's ratio, were determined using the methods described in Refs. [33-35] (see SI part II for calculation details). These parameters were calculated at $0 \mathrm{~K}$ and are listed in Table 2. The temperature-dependent CRSS, denoted as $\tau_{\mathrm{c}, \mathrm{s}}^{T}$ for the shuffle-set dislocation and $\tau_{\mathrm{c}, \mathrm{g}}^{T}$ for the glide-set dislocation, can then be evaluated from Equation (5) with a geometric or numerical method. These two types of dislocations are in competition, and the one with a lower CRSS dominates the deformation at a given temperature and shear stress. Therefore, the CRSS of a material can be determined using

$\widehat{\tau}_{\mathrm{c}}^{T}=\min \left(\tau_{\mathrm{c}, \mathrm{s}}^{T}, \tau_{\mathrm{c}, \mathrm{g}}^{T}\right)$.

For zinc-blende structured polycrystalline materials, the pre-factors in Schmid's law and Tabor's law are 3.1 [36,37] and 2.74 [29], respectively. Therefore, Equation (8) for zinc-blende structured covalent materials can be rewritten as

$H_{T}=8.5 \widehat{\tau}_{c}^{T}$.

Fig. 2a displays the calculated Vickers hardness at $300 \mathrm{~K}$ for typical zinc-blende structured covalent materials as compared with the experimental data [4], which are highly consistent. The calculated temperature-dependent Vickers hardness of diamond, Si, and Ge are shown in Fig. 2b-d, respectively (see Fig. S3 for the temperature-dependent lattice constant, shear moduli, and Poisson's ratio) and are in good agreement with the experimental hardness values measured over a wide temperature range [1,11-14]. In addition, the temperature-dependent Vickers hardnesses of polar covalent materials, cubic $\mathrm{BN}$ and $\mathrm{SiC}$, are plotted in Fig. $\mathrm{S} 4$ and are also found to be in good accordance with the experimental values. Overall, the hardness decreases with increasing temperature for pure covalent materials (diamond, $\mathrm{Si}$, and $\mathrm{Ge}$ ) and for polar covalent materials ( $\mathrm{SiC}$ and $\mathrm{BN}$ ). At a critical temperature, the hardness abruptly decreases with increasing temperature. By evaluating the dominant dislocations at different temperatures, it was found that the primary dislocation changed from shuffle-set dislocations to glide-set dislocations at this critical temperature. Moreover, the calculated transition temperatures where the shuffle-set dislocation-

Table 1 Geometric parameters for $\frac{1}{6}<112>$ glide-set $90^{\circ}$ partial and $\frac{1}{2}<110>$ shuffle-set perfect dislocations

\begin{tabular}{cccccccc}
\hline Dislocation type & $\beta$ & $b$ & $h$ & $r$ & $\rho_{\mathrm{m}}\left(\mathrm{m}^{-2}\right)$ & $\lambda_{\mathrm{b}}(\mathrm{nm})$ & $\dot{\varepsilon}\left(\mathrm{s}^{-1}\right)$ \\
\hline$\frac{1}{2}<110>$ shuffle-set & $0^{\circ}$ & $\sqrt{2} a / 2$ & $\sqrt{6} a / 4$ & $0.9 b$ & $0.3 \times$ & $10^{8}$ & 100 \\
$\frac{1}{6}<112>$ glide-set & $90^{\circ}$ & $\sqrt{6} a / 6$ & $\sqrt{6} a / 4$ & $0.3 b$ & $0.3 \times 10^{14}$ & 100 & $10^{-4}$ \\
\hline
\end{tabular}


Table 2 Calculated lattice constants, shear moduli, $a^{3} G$, Poisson's ratios, Debye frequencies at $0 \mathrm{~K}$, and shuffle-set to glide-set transition temperatures for selected zinc-blende structured covalent materials. Calculated hardness values are given at each temperature ( 0 and $300 \mathrm{~K})$ in comparison with the experimental values.

\begin{tabular}{|c|c|c|c|c|c|c|c|c|c|c|}
\hline Phase & $a(\AA)$ & $\begin{array}{c}G \\
(\mathrm{GPa})\end{array}$ & $\begin{array}{c}a^{3} G \\
\left(\times 10^{-18} \mathrm{~J}\right)\end{array}$ & $v$ & $\begin{array}{c}v_{\mathrm{D}} \\
(\mathrm{THz})\end{array}$ & $\begin{array}{c}H_{0} \\
(\mathrm{GPa})\end{array}$ & $H_{300 \mathrm{~K}}(\mathrm{GPa})$ & $H_{\exp }(\mathrm{GPa})$ & $\begin{array}{l}T_{\mathrm{s}-\mathrm{g}} \\
(\mathrm{K})\end{array}$ & $\begin{array}{c}T_{\text {s-g }} \exp \\
(\mathrm{K})\end{array}$ \\
\hline Diamond & 3.57 & 521 & 23.7 & 0.07 & 39.3 & 94.7 & 85.6 & $60-150^{\mathrm{a}}$ & 1402.6 & $1450^{\mathrm{e}}$ \\
\hline $\mathrm{Si}$ & 5.47 & 64.2 & 10.5 & 0.21 & 15.1 & 10.9 & 8.9 & $11.3^{\mathrm{b}}$ & 676.8 & $650^{f}$ \\
\hline $\mathrm{Ge}$ & 5.78 & 45 & 8.7 & 0.19 & 8.3 & 7.8 & 6.1 & $7.2^{\mathrm{c}}$ & 560.2 & $600^{g}$ \\
\hline AlAs & 5.73 & 43.86 & 8.3 & 0.23 & 11.3 & 7.3 & 5.6 & $5^{\mathrm{d}}$ & 542.8 & \\
\hline AlP & 5.51 & 52 & 8.7 & 0.24 & 12.9 & 10.7 & 8.9 & $9.4^{\mathrm{d}}$ & 722.5 & \\
\hline $\mathrm{AlSb}$ & 6.23 & 32 & 7.7 & 0.23 & 9.8 & 5.3 & 4 & $4^{\mathrm{d}}$ & 509.7 & \\
\hline BAs & 4.82 & 129.4 & 14.5 & 0.13 & 21.4 & 23.1 & 19.8 & $19^{\mathrm{b}}$ & 886.4 & \\
\hline $\mathrm{BN}$ & 3.63 & 390 & 18.7 & 0.11 & 34.2 & 70.2 & 62.0 & $46-80^{a}$ & 1125.5 & $1000^{\mathrm{h}}$ \\
\hline BP & 4.55 & 168 & 15.8 & 0.11 & 24.0 & 30.2 & 26.1 & $31^{\mathrm{a}}$ & 957.9 & \\
\hline GaAs & 5.76 & 43.68 & 8.3 & 0.22 & 7.7 & 7.3 & 5.8 & $7.5^{\mathrm{b}}$ & 550.4 & $370^{\mathrm{f}}$ \\
\hline $\mathrm{GaP}$ & 5.53 & 54 & 9.1 & 0.22 & 10.8 & 9.1 & 7.2 & $9.5^{\mathrm{d}}$ & 597.4 & $350^{f}$ \\
\hline $\mathrm{GaSb}$ & 6.22 & 32.2 & 7.7 & 0.21 & 6.4 & 6.7 & 5.4 & $4.5^{\mathrm{d}}$ & 625.7 & \\
\hline InAs & 6.21 & 28.3 & 6.8 & 0.26 & 6.2 & 4.5 & 3.3 & $3.8^{\mathrm{d}}$ & 463.6 & \\
\hline InP & 6 & 34.1 & 7.4 & 0.26 & 9.4 & 5.5 & 4.1 & $5.4^{\mathrm{d}}$ & 498.7 & $420^{\mathrm{f}}$ \\
\hline InSb & 6.65 & 22.28 & 6.6 & 0.25 & 5.0 & 3.6 & 2.7 & $2.2^{\mathrm{d}}$ & 445.9 & $300^{\mathrm{f}}$ \\
\hline $\mathrm{SiC}$ & 4.38 & 198.74 & 16.7 & 0.14 & 24.1 & 33.4 & 29.4 & $26-37^{a}$ & 1076.4 & $650^{\mathrm{i}}$ \\
\hline
\end{tabular}

a), e), i) Vickers hardness from Ref. [38], Ref. [10] and Ref. [39], respectively; b), f) Knoop hardness from Ref. [40] and Ref. [1], respectively; c), g) Vickers hardness from Ref. [41] and Ref. [13], respectively; d), h) Knoop hardness from Ref. [42] and Ref. [43], respectively.

controlled deformation changed to a glide-set dislocation-controlled deformation $\left(T_{s^{-}}\right)$were 1402.6, 676.8, and $560.2 \mathrm{~K}$ for diamond, $\mathrm{Si}$, and $\mathrm{Ge}$, respectively, which are comparable to the experimental values, $1450 \mathrm{~K}$ for diamond [10], $650 \mathrm{~K}$ for Si [1], and $600 \mathrm{~K}$ for Ge [13].

At low temperatures, the dislocation motions caused by thermal activation can be ignored. In this case, the Vickers hardness determined from Equations (5-9) for a zinc-blende structured covalent material can be determined by the shear modulus and Poisson's ratio, and Equation (8) can be simplified as

$H_{0}=k_{0}(v) G$,

where $k_{0}(v)$ is a proportional coefficient related to Poisson's ratio. Fig. 3 shows the Vickers hardness map at $0 \mathrm{~K}$ constructed from Equation (8), where it is obvious that both the shear modulus and Poisson's ratio modulate the Vickers hardness. As shown in Fig. 3b, the Vickers hardness increases linearly with an increase in the shear modulus, and $k_{0}(v)$ decreases slightly with an increase in Poisson's ratio. Compared with the shear modulus, Poisson's ratio has a smaller effect on the Vickers hardness (Fig. 3c). Overall, a high shear modulus and a low
Poisson's ratio are essential for (super)hard materials, which is consistent with Chen's deduction [4]. In addition, the proportional coefficient $k_{0}(v)$ varies within the narrow range of $0.14-0.19$ when $v$ is in the range of $0-0.3$ (inset of Fig. 3a). By fitting the relationship between $k_{0}(v)$ and $v$ as shown in Fig. 3a, Equation (8) can be rewritten as

$H_{0}=\left(0.18+0.05 v-0.51 v^{2}\right) G$

Previously, some semi-empirical hardness models relate hardness to materials' shear modulus using the proportional coefficients of 0.12 [1], 0.151 [4], and 0.147 [5], obtained by fitting the experimental data. These values agree well with the $k_{0}(v)$ determined in the current work, verifying the effectiveness of the calculation of hardness on the basis of dislocation dynamics.

At elevated temperatures, the thermally activated dislocation motions are no longer negligible. From Equation (5), $\tau_{\mathrm{c}}^{T} / G$ is clearly correlated with Poisson's ratio, temperature, and the materials-related parameter $a^{3} G$, which, combined with Equation (8), gives the temperature-dependent Vickers hardness as 

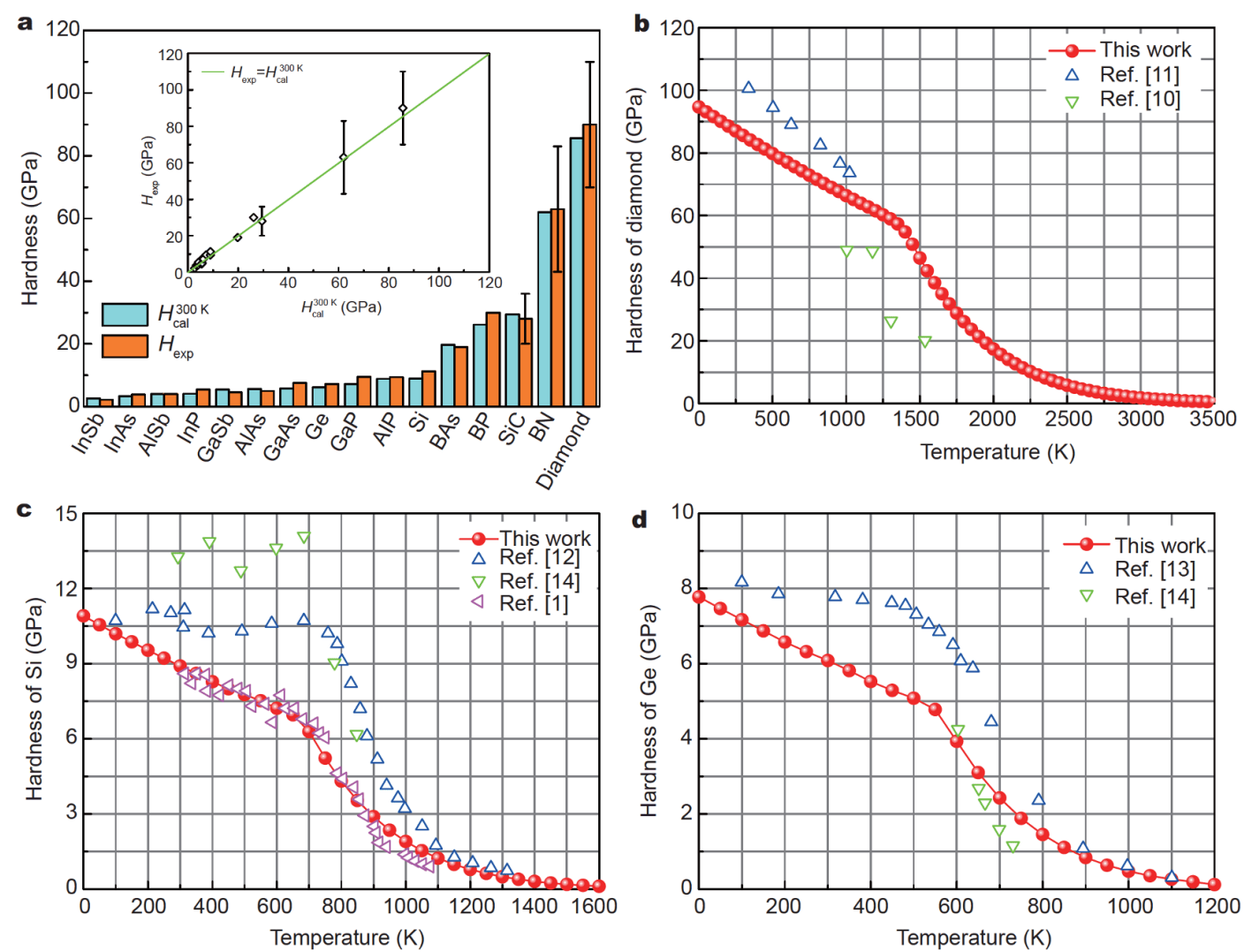

Figure 2 Calculated Vickers hardness compared with experimental values. (a) Comparison of Vickers hardness values from the current work and the experimental results at $300 \mathrm{~K}$. (b-d) Calculated temperature-dependent Vickers hardness for diamond, Si, and Ge in comparison with experimental data $[1,10-14]$.

$H=k\left(T, a^{3} G, v\right) G$,

where the proportional coefficient $k$ is a function of $v, T$, and $a^{3} G$. The thermal effects and the material's nonelastic properties are therefore included in $k$. Note that $k=H / G$ can be used as a normalized hardness with respect to a material's shear modulus.

Fig. 4 shows $k$ as a function of $v, T$, and $a^{3} G$. By considering the general range of $\rho_{\mathrm{m}}, \lambda_{\mathrm{b}}$, and $\dot{\varepsilon}$ for the materials of interest (such as those listed in Table 1), the estimated values of the second logarithm in Equation (5), $\ln \left(\frac{\rho_{\mathrm{m}} b \lambda_{\mathrm{b}} v_{\mathrm{D}}}{\dot{\varepsilon}}\right)$, were found to vary over a relatively narrow range for different zinc-blende structured covalent materials (Fig. S5). Therefore, the fixed values of 18.8 and 32.1 were used in the calculation of shuffle-set and glideset dislocations, respectively. A similar simplification was previously reported [44]. As shown in Fig. $4 a, k$ decreases with increasing temperature for a given set of Poisson's ratio and $a^{3} G$, indicating that softening occurs at higher temperatures. Furthermore, for a fixed Poisson's ratio, larger values of $a^{3} G$ result in a slower decrease in $k$, indicating that materials with a larger $a^{3} G$ are more difficult to soften with increasing temperature. The calculated transition temperature $T_{\mathrm{s}-\mathrm{g}}$, where shuffle-set dislocation control switches to glide-set dislocation control, is shown in Fig. 4b. $T_{s-g}$ increases linearly with $a^{3} G$ at a fixed Poisson's ratio, and increases to a higher temperature with a larger Poisson's ratio. Therefore, it is accurate to classify $a^{3} G$ as an intrinsic index for zinc-blende structured covalent materials to measure the resistance to softening at elevated temperatures, which is consistent with previously reported semi-empirical results [45].

In addition to the above-mentioned intrinsic properties that determine the hardness of a material, other factors, such as the dislocation characteristics, microstructure, and loading conditions of the sample, can also demonstrate a profound impact on the hardness. In the current 

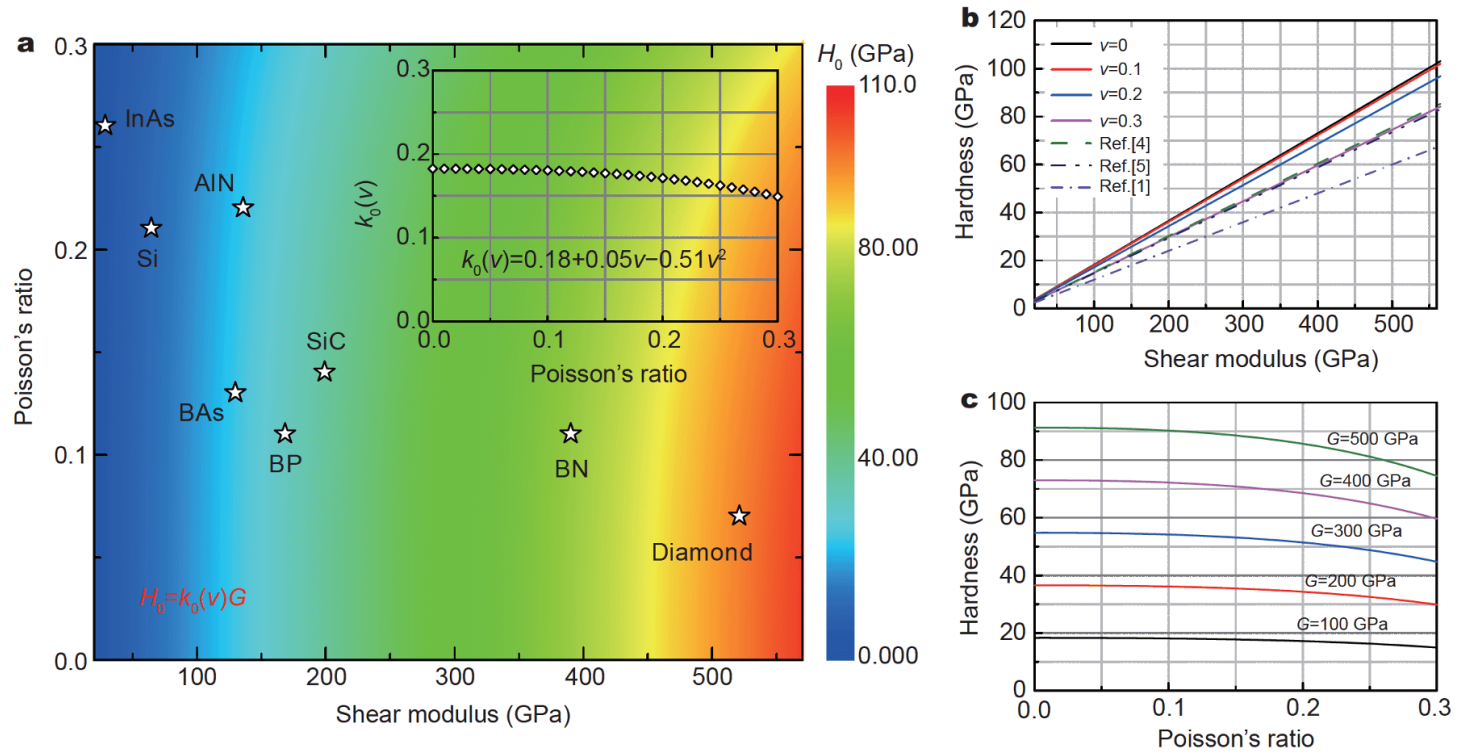

Figure 3 Effect of shear modulus and Poisson's ratio on hardness of zinc-blende structured covalent materials at $0 \mathrm{~K}$. (a) Calculated Vickers hardness map as a function of shear modulus and Poisson's ratio. (b) Effect of shear modulus on material hardness with different Poisson's ratios. (c) Effect of Poisson's ratio on material hardness with different shear moduli.
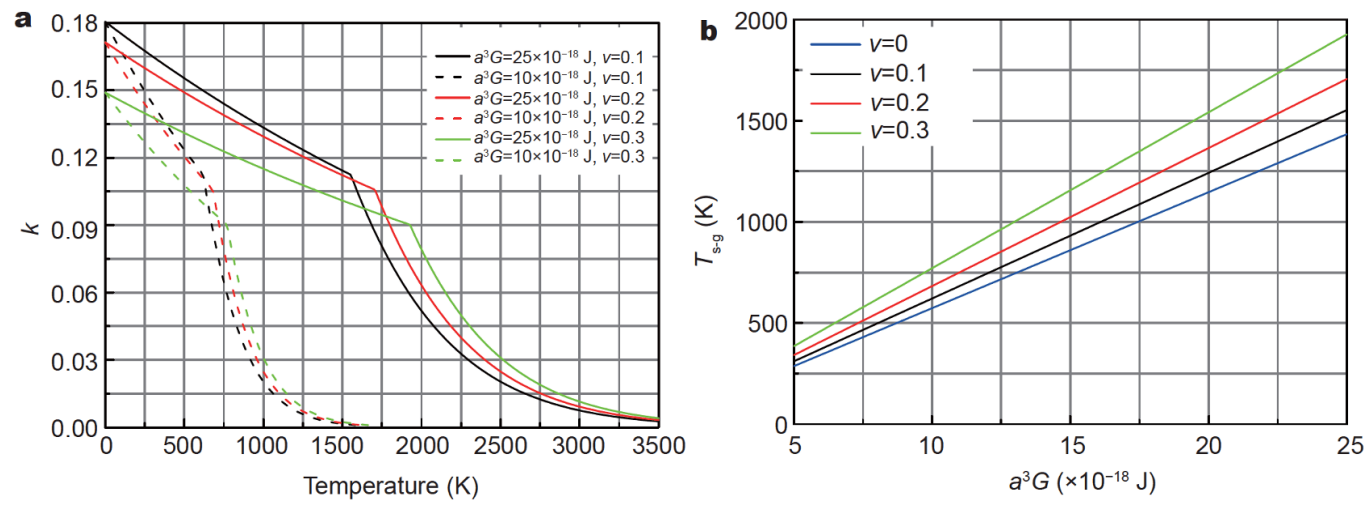

Figure 4 Temperature effect on Vickers hardness of zinc-blende structured covalent materials. (a) The effects of temperature, $a^{3} G$, and Poisson's ratio on $k$. (b) The effect of $a^{3} G$ and Poisson's ratio on the transition temperature $T_{\mathrm{s}-\mathrm{g}}$.

model, as in Equation (5), the dislocation density and strain rate are included so that their effects on the hardness of a material can be studied. In addition, the influence of grain boundaries on hardness can be studied using the Hall-Petch effect [28]. Therefore, these additional effects can be easily accounted for in the current dislocation-based hardness model. For example, the hardness varies with the density of mobile dislocations: the larger the dislocation density, the lower the hardness (Fig. S6a). The effects of loading speed (Fig. S6b) and the grain boundary (Fig. S6c) on hardness were also in- vestigated.

\section{CONCLUSIONS}

In summary, a temperature-dependent Vickers hardness model was developed based on the dislocation theory for zinc-blende structured covalent materials. At low temperatures, the Vickers hardness is primarily controlled by Poisson's ratio and the shear modulus, with the latter playing a more dominant role. At elevated temperatures, the deformation mechanism changes from shuffle-set dislocation control to glide-set dislocation control, and 
the Vickers hardness is further affected by the temperature and a material-related parameter, $a^{3} G$. Materials with a larger value of $a^{3} G$ are less prone to softening at elevated temperatures. These findings help to elucidate the physics of hardness and provide direct guidance for the design of superhard materials, especially at high temperatures.

\section{Received 1 October 2020; accepted 19 January 2021; published online 26 March 2021}

1 Gilman JJ. Chemistry and Physics of Mechanical Hardness. New Jersey: John Wiley \& Sons, 2009: 261-298

2 Liu AY, Cohen ML. Prediction of new low compressibility solids. Science, 1989, 245: 841-842

3 Teter DM. Computational alchemy: The search for new superhard materials. MRS Bull, 1998, 23: 22-27

4 Chen XQ, Niu H, Li D, et al. Modeling hardness of polycrystalline materials and bulk metallic glasses. Intermetallics, 2011, 19: 12751281

5 Jiang X, Zhao J, Jiang X. Correlation between hardness and elastic moduli of the covalent crystals. Comput Mater Sci, 2011, 50: 22872290

6 Gao F, He J, Wu E, et al. Hardness of covalent crystals. Phys Rev Lett, 2003, 91: 015502

7 Simůnek A, Vackár J. Hardness of covalent and ionic crystals: First-principle calculations. Phys Rev Lett, 2006, 96: 085501

8 Li K, Wang X, Zhang F, et al. Electronegativity identification of novel superhard materials. Phys Rev Lett, 2008, 100: 235504

9 Guo X, Li L, Liu Z, et al. Hardness of covalent compounds: Roles of metallic component and d valence electrons. J Appl Phys, 2008, 104: 023503

10 Weidner DJ, Wang Y, Vaughan MT. Strength of diamond. Science, 1994, 266: 419-422

11 Novikov NV, Sirota YV, Mal'nev VI, et al. Mechanical properties of diamond and cubic $\mathrm{BN}$ at different temperatures and deformation rates. Diamond Related Mater, 1993, 2: 1253-1256

12 Suzuki T, Ohmura T. Ultra-microindentation of silicon at elevated temperatures. Philos Mag A, 1996, 74: 1073-1084

13 Gerk AP. Germanium microhardness and the correlation of indentation creep with dislocation velocity. Philos Mag, 1975, 32: 355-365

14 Trefilov V, Mil'Man YV. Aspects of the plastic deformation of crystals with covalent bonds. Soviet Phys Doklady, 1964, 8: 1240

15 Mukhanov VA, Kurakevych OO, Solozhenko VL. Hardness of materials at high temperature and high pressure. Philos Mag, 2009, 89: 2117-2127

16 Ceder G. Computational materials science: Predicting Properties from Scratch. Science, 1998, 280: 1099-1100

$17 \mathrm{Hu} \mathrm{W}$, Wen B, Huang Q, et al. Role of plastic deformation in tailoring ultrafine microstructure in nanotwinned diamond for enhanced hardness. Sci China Mater, 2017, 60: 178-185

$18 \mathrm{Wu} \mathrm{Y}$, Zhang $\mathrm{Y}$, Zhang $\mathrm{S}$, et al. The rise of plastic deformation in boron nitride ceramics. Sci China Mater, 2021, 64: 46-51

19 Peierls R. The size of a dislocation. Proc Phys Soc, 1940, 52: 34-37

20 Nabarro FRN. Dislocations in a simple cubic lattice. Proc Phys Soc, 1947, 59: 256-272

21 Nabarro FR, Duesbery MS. Dislocations in Solids. Amsterdam: Elsevier, 2002

22 Tian Y. Rise of correlated dislocations in nanotwinned metals against fatigue. Sci China Mater, 2018, 61: 127-128

23 Xiao J, Yang $\mathrm{H}, \mathrm{Wu} \mathrm{X}$, et al. Dislocation behaviors in nanotwinned diamond. Sci Adv, 2018, 4: eaat8195

24 Wen $\mathrm{B}, \mathrm{Xu} \mathrm{B}$, Wang $\mathrm{Y}$, et al. Continuous strengthening in nanotwinned diamond. npj Comput Mater, 2019, 5: 117

25 Xiao J, Wen B, Xu B, et al. Intersectional nanotwinned diamondthe hardest polycrystalline diamond by design. npj Comput Mater, 2020, 6: 119

26 Xu B, Tian Y. Superhard materials: Recent research progress and prospects. Sci China Mater, 2015, 58: 132-142

27 Luo K, Zhang Y, Yu D, et al. Small onion-like BN leads to ultrafine-twinned cubic BN. Sci China Mater, 2019, 62: 1169-1176

28 Hirth JP, Lothe J, Mura T. Theory of Dislocations (2nd ed.). Florida: Wiley, 1983

29 Tabor D. The Hardness of Metals. New York: Oxford university press, 2000: 553

30 Kolar HR, Spence JCH, Alexander H. Observation of moving dislocation kinks and unpinning. Phys Rev Lett, 1996, 77: 40314034

31 Blumenau AT, Heggie MI, Fall CJ, et al. Dislocations in diamond: Core structures and energies. Phys Rev B, 2002, 65: 205205

32 Yang $\mathrm{H}$, Xiao J, Yao Z, et al. Homogeneous and heterogeneous dislocation nucleation in diamond. Diamond Related Mater, 2018, 88: $110-117$

33 Shao T, Wen B, Melnik R, et al. Temperature dependent elastic constants for crystals with arbitrary symmetry: Combined first principles and continuum elasticity theory. J Appl Phys, 2012, 111: 083525

34 Wen B, Shao T, Melnik R, et al. Temperature and pressure dependent geometry optimization and elastic constant calculations for arbitrary symmetry crystals: Applications to $\mathrm{MgSiO}_{3}$ perovskites. J Appl Phys, 2013, 113: 103501

35 Feng X, Xiao J, Melnik R, et al. Mechanical and thermal properties of $\gamma-\mathrm{Mg}_{2} \mathrm{SiO}_{4}$ under high temperature and high pressure conditions such as in mantle: A first principles study. J Chem Phys, 2015, 143: 104503

36 Praveen Sekhar A, Nandy S, Ray KK, et al. Hardness-yield strength relation of Al-Mg-Si alloys. IOP Conf Ser-Mater Sci Eng, 2018, 338: 012011

37 Stoller RE, Zinkle SJ. On the relationship between uniaxial yield strength and resolved shear stress in polycrystalline materials. J Nucl Mater, 2000, 283-287: 349-352

38 Brazhkin VV, Lyapin AG, Hemley RJ. Harder than diamond: Dreams and reality. Philos Mag A, 2002, 82: 231-253

39 Milman YV, Chugunova SI, Goncharova IV, et al. Temperature dependence of hardness in silicon-carbide ceramics with different porosity. Int J Refractory Met Hard Mater, 1999, 17: 361-368

40 Williams ML. CRC Handbook of Chemistry and Physics, 76th edition. Occupational Environ Med, 1996, 53: 504

41 Szymański A, Szymański JM. Hardness Estimation of Minerals, Rocks and Ceramic Materials. Materials Science Monographs. Published Place: Amsterdam: Elsevier, 1989, 49: IX-330

42 Sung CM, Sung M. Carbon nitride and other speculative superhard materials. Mater Chem Phys, 1996, 43: 1-18

43 Wheeler JM, Michler J. Invited article: Indenter materials for high temperature nanoindentation. Rev Sci Instruments, 2013, 84: 101301

44 Basinski ZS. Thermally activated glide in face-centred cubic metals and its application to the theory of strain hardening. Philos Mag, 1959, 4: 393-432 
45

Siethoff $\mathrm{H}$. Correspondence of the plasticity of rocksalt structure ceramics and tetrahedrally coordinated semiconductors. J Appl Phys, 2003, 94: 3128-3134

Acknowledgements This work was supported by the National Natural Science Foundation of China (51925105, 51771165, and 51525205), the National Magnetic Confinement Fusion Energy Research Project of China (2015GB118001), the US National Science Foundation (NSF, EAR-1361276), and the National Key R\&D Program of China (YS2018YFA070119).

Author contributions Wen B conceived the project. Feng X and Wen $B$ performed the model construction and calculations. Feng $X$, Wen $B$, and $\mathrm{Xu} \mathrm{B}$ wrote the paper. Xiao J, Zhao J, Wang Y, and Tian Y helped perform the analysis and provided constructive discussions.

Conflict of interest The authors declare that they have no conflict of interest.

Supplementary information Experimental details and supporting data are available in the online version of the paper.

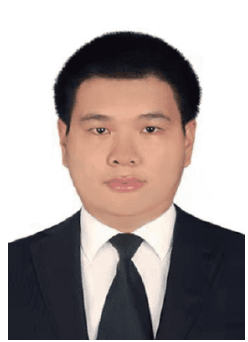

Xing Feng is currently a $\mathrm{PhD}$ candidate at the State Key Laboratory of Metastable Materials Science and Technology, Yanshan University. He obtained his bachelor's degree from the School of Materials Science and Engineering, Shijiazhuang Tiedao University in 2013. His current research focuses on the relationship between the macrostrength and microstructure of materials.

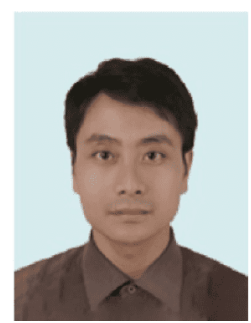

Bin Wen is a professor at the College of Materials Science and Engineering, Yanshan University. He received his $\mathrm{PhD}$ from Dalian University of Technology in 2006. From 2008 to 2010 , he was engaged in postdoctoral research at Wilfred Laurier University and Tohoku University. He joined the State Key Laboratory of Metastable Materials Science and Technology, Yanshan University in 2010. His research interests focus on the micro-mechanism of mechanical properties of materials.

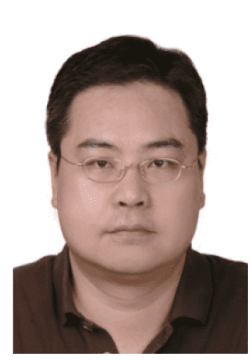

Bo Xu is a professor at the College of Materials Science and Engineering, Yanshan University. $\mathrm{He}$ received his $\mathrm{PhD}$ from the University of Nebraska-Lincoln (2002). After completing his postdoctoral work at the University of Maryland, College Park, he joined the State Key Laboratory of Metastable Materials Science and Technology, Yanshan University in 2006. His research interests include superhard materials and thermoelectric materials.

\section{闪锌矿结构共价材料的高温硬度}

冯幸 ${ }^{1}$, 肖建伟 ${ }^{1}$, 温斌 ${ }^{*}$, 赵纪军 ${ }^{2}$, 徐波 $^{1^{*}}$, 王雁宾 ${ }^{3}$, 田永君 ${ }^{1}$

摘要 共价材料高温硬度的研究, 不仅具有基本的科学意义, 而且 具有非常重要的应用价值. 基于位错理论, 本文提出了一个针对闪 锌矿结构共价材料高温维氏硬度的理论模型. 在忽略温度效应时, 硬度仅与泊松比和剪切模量有关, 且剪切模量的影响较大. 随温度 升高, 硬度下降, 当大于某一临界温度时, 其下降趋势陡然增加, 该 下降趋势的突变主要来源于控制位错滑移的位错由拖拉面位错向 滑移面位错转变. 研究结果还表明, 材料本征参数 $a^{3} G$ 可以用来表 征材料抗升温软化的能力. 此外, 本研究的计算结果与实验结果很 好吻合. 本研究不仅可以解释硬度的物理本质, 也可为超硬材料的 设计提供指导. 